regulation of oxidative metabolism and potential roles in development of systemic lupus erythematosus (SLE).

Methods CD4 + T lymphocytes isolated from 97 SLE patients, 30 RA patients, 10 AS patients and 20 healthy donors were used in our in vitro experiments. Total RNA of CD4 + T lymphocytes isolated from 4 SLE patients and 3 healthy donors were used to perform small RNA sequencing. Total RNA of CD4 + T lymphocytes transfected with synthetic tRF-3009 or negative control (a random sequence, single strand) were used to perform next generation sequencing. CD4 + T lymphocytes isolated from healthy donors were transfected with tRF-3009/negative control or tRF-3009 siRNA/si-NC in vitro, with/without IFN-alpha treatment, to analyze OCRROS and ATP concentration. Real-time qRT-PCR was performed to analyze expression of tRF-3009 and related gene expressions.

Results We have identified a series of tRFs expressed abnormally in SLE CD4 + T lymphocytes. Interestingly, almost all up regulated tRFs were from 3 end of mature tRNA while down regulated ones were from 3 end of tRNA precursors. We have found that expression of tRF-3009 was corelated with lupus nephritis (LN) and urine protein (PRO). The expression of tRF-3009 could be induced by IFN-alpha treatment in vitro. Knockdown of tRF-3009 by siRNA could rescue the metabolism change of $\mathrm{CD} 4+\mathrm{T}$ lymphocytes induced by IFN-alpha. Transfection of tRF-3009 alone could up regulate oxidative phosphorylation of CD4 + T lymphocytes in vitro.

Conclusions This study identified a series of abnormally expressed tRFs existed in CD4 + T lymphocytes isolated from SLE patients. One of these small RNAs, tRF-3009 also enriched in kidney and corelated with occurrence of lupus nephritis. tRF-3009 anticipated in metabolism regulation, may play important roles in SLE pathogenesis.

Funding Source(s): The National Key Research and Development Program of China(2016YFC0903900 and 2017YFC0909000)

\section{PREDICTORS OF HYDROXYCHLOROQUINE TREATMENT ADHERENCE AMONG PEOPLE WITH LUPUS}

Karin Tse ${ }^{*}$, Rossi Paola Daly. Lupus Foundation of America

\subsection{6/lupus-2019-Ism.232}

Background Treatment adherence is associated with better disease control, improved morbidity and mortality and increased quality of life. ${ }^{1}$ Studies examining treatment adherence predictors have varied in examined characteristics and results. This study analyzes demographic and socioeconomic factors, perceptions, and experiences influencing hydroxychloroquine (HCQ) treatment adherence among people with lupus. HCQ has long been used to treat lupus, and has been shown to reduce damage accrual and has protective effects on diseases such as diabetes and hyperlipidemia as well as overall survival in people with lupus. ${ }^{2}$

Methods Cross-sectional study data was obtained through the 2015-2016 Lupus Needs Survey, an online national needs assessment among 3022 adults self-reporting a lupus diagnosis. Univariate analyses were conducted using chi-square and simple logistic regressions among 21 variables, which were subsequently dichotomized. Variables with rates of missing values $>15 \%$ of total sample were removed in further analysis.
Abstract 232 Table 1 Results of Binary Logistic Model of Predictors of HCQ Treatment Adherence

\begin{tabular}{|c|c|c|c|}
\hline & Odds Ratio & $\begin{array}{c}95 \% \\
\text { Confidence } \\
\text { Interval }\end{array}$ & $p$-value \\
\hline Black & 0.35 & $0.18-0.67$ & $0.002^{*}$ \\
\hline $\begin{array}{l}\text { Other (mixed, American Indian/Alaskan Native, } \\
\text { Pacific Islander, or Middle Eastern) }\end{array}$ & 0.35 & $0.14-0.85$ & $0.021^{*}$ \\
\hline Moderate severity of lupus at diagnosis & 0.57 & $0.31-1.03$ & 0.061 \\
\hline HCQ improves/helps treat my symptoms & 2.49 & $1.25-4.92$ & $0.009^{*}$ \\
\hline Positive impression of HCQ & 2.14 & $1.08-4.24$ & $0.029^{*}$ \\
\hline Rheumatologist as primary doctor treating lupus & 2.0 & $1.01-3.95$ & $0.046^{*}$ \\
\hline
\end{tabular}

Next, 13 variables with a $\mathrm{p}$-value $<0.20$ were entered into a binary logistic model using backwards stepwise selection.

Results 2028 individuals reported currently taking HCQ with $1581(78.0 \%)$ individuals reporting taking the medication exactly as prescribed all of the time, 385 (19.0\%) reporting some of the time, and $62(3.1 \%)$ reporting sometimes or never taking HCQ as prescribed. People with lupus were twice as likely to adhere to HCQ if they reported having a rheumatologist as the primary doctor treating their lupus $(p=0.046, O R=2.00)$ and more than twice as likely if they reported having a positive impression of HCQ $(p=0.029$, $\mathrm{OR}=2.14$ ) or experienced improvement in treating their symptoms using HCQ $(p=0.009, \mathrm{OR}=2.49)$. Notably, African Americans and those indicating race as Other (mixed, American Indian/Alaskan Native, Pacific Islander or Middle Eastern) were $65 \%$ less likely to adhere to HCQ treatment $(p=0.002$, $\mathrm{OR}=0.35 ; \mathrm{p}=0.021, \mathrm{OR}=0.35$, respectively).

Conclusions The study confirms the importance of experiential factors such as medication impression and patient-reported symptom improvement in HCQ treatment adherence. Additionally, results highlight the potential significance of having a specialist as the primary lupus doctor. This may be due to rheumatologists higher awareness of the value of HCQ for lupus patients compared to other providers. Further exploration is needed on cultural factors negatively influencing treatment adherence among certain racial groups, particularly African Americans and Other racial groups (mixed, American Indian/Alaskan Native, Pacific Islander or Middle Eastern). Treatment adherence is particularly important in lupus given the complex nature of the disease and fluctuation of disease activity, which may be exacerbated by poor adherence.

Funding Source(s): UCB Pharma funded study data collection.

\section{AUTOANTIBODY-POSITIVE HEALTHY INDIVIDUALS CONSTRAIN T CELL PATHWAYS TO REGULATE AUTOIMMUNE DISEASE}

'Samantha Slight-Webb, 'Aleksandra Bylinska, ${ }^{1}$ Miles Smith, ${ }^{1}$ Rufei Lu, ${ }^{1}$ Hua Chen, ${ }^{1}$ Krista Bean, ${ }^{1}$ Melissa Munroe, ${ }^{2}$ Holden Maecker, ${ }^{2}$ Paul J Utz, ${ }^{1}$ Joan T Merrill, ${ }^{3}$ Eliza Chakravarty, ${ }^{1}$ Cristina Arriens, ${ }^{1}$ Joel Guthridge, ${ }^{1}$ Judith A James*. ' Oklahoma Medical Research Foundation; ${ }^{2}$ Stanford University; ${ }^{3}$ Oklahoma Medical Research Foundation; University of Oklahoma

\subsection{6/lupus-2019-Ism.233}

Background Anti-nuclear antibody (ANA) positivity is a principal feature of individuals with an autoimmune disease, yet up to one in five healthy individuals are ANA-positive (ANA+) 
A

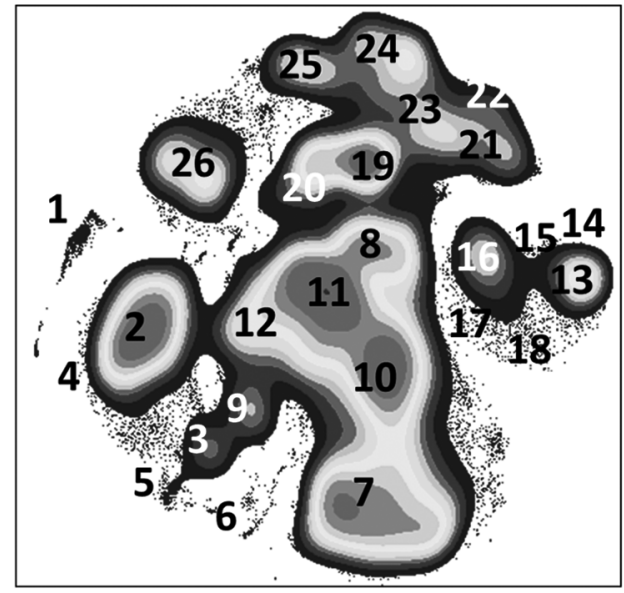

1) Plasmacytoid DCs

2) Monocytes (Classical)

3) Non-Classical Monocytes

4) Intermediate Monocytes

5) Dendritic Cells

6) $\mathrm{CD} 11 \mathrm{c}+\mathrm{B}$ cells

7) CD4+ Naïve/Effector T cells

8) CXCR5+CD4+ Memory T cells

9) CD27-CD4+Memory $T$ cells

10) Central Memory CD4+ T cells

11) CXCR3+ Memory CD4+ T cells

12) Effector Memory CD4+ T cells

13) Memory $B$ cells
14) Plasmablasts

15) Non-switched Memory B cells

16) Naïve $B$ cells

17) Transitional $B$ Cells

18) DN B cells

19) CD57+ NK Cells

20) CD57- NK Cells

21) $\mathrm{CD} 57+\mathrm{CD} 8+\mathrm{T}$ cells

22) TCRgd T Cells

23) CD8+ Effector T cells

24) DN T cells

25) CD8+ Memory T cells

26) CD8+ Naïve T Cells

B

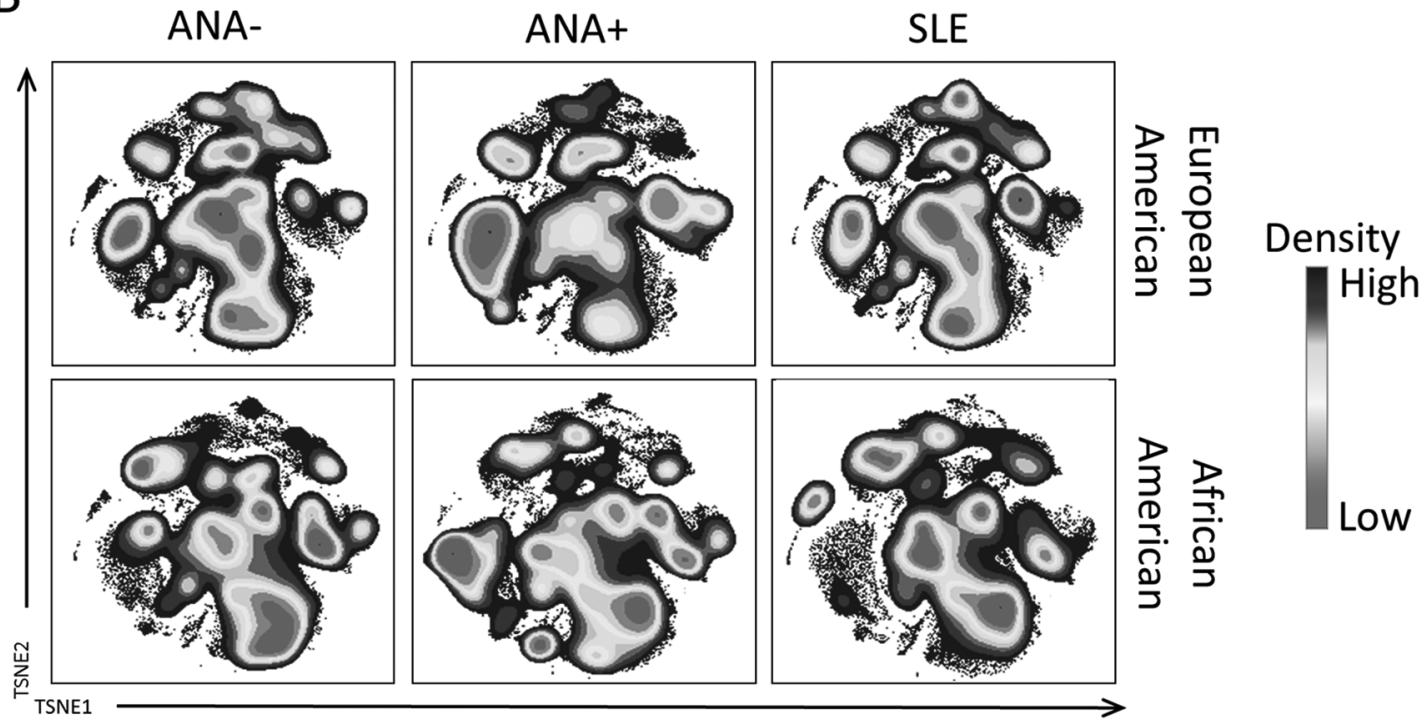

C

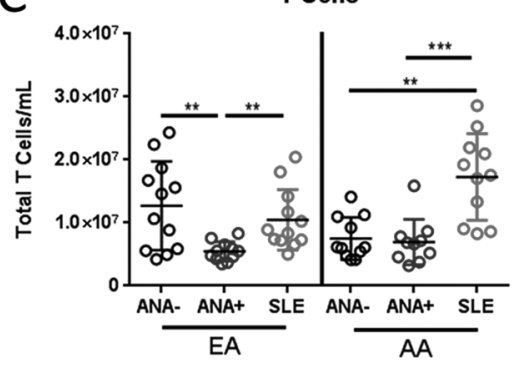

CD4+ T Cells

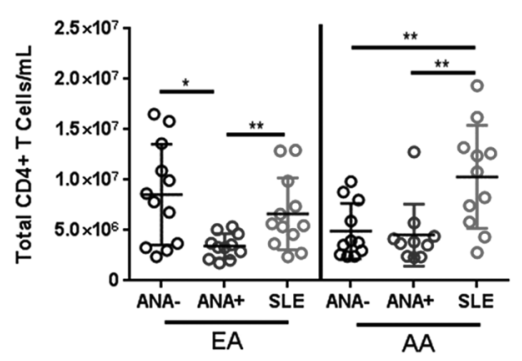

CD8+ T Cells

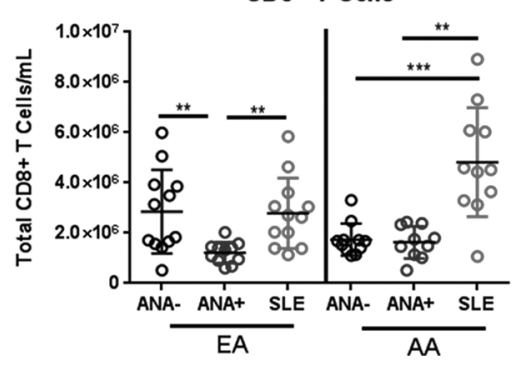

Abstract 233 Figure 1 Calculated cell numbers indicate elevated T cells in SLE patients and suppressed T cells in EA ANA+ healthy individuals. 20 cell surface marker expression is shown using dimensionality reduced t-SNE plots from PBMC data (110,00 cells) derived from 72 samples. (A) A density map is shown depicting the density of cells and are numbered according to phenotypic subset. (B) Density maps depicting European and African American ANA-, ANA+ and SLE patient PBMC t-SNE plots created using all 33 surface markers are plotted. All plots were derived from cumulative data from 12 individuals per group. (C) Cells numbers were calculated from cell subsets using frequencies and total cell counts. Cell numbers for T cells, CD4+ T cells, and CD8+ T cells are shown. ${ }^{*} p<0.05,{ }^{*} p<0.01,{ }^{* * *} p<0.001$ 
and will never develop overt disease. Understanding differences in immune cell physiology between ANA+healthy individuals and individuals with clinical SLE remains a critical goal in the understanding of SLE pathogenesis across ethnicities.

Methods Blood specimens and information on disease activity were collected from European (EA) and African American (AA) individuals classified and matched in groups as ANAhealthy controls $(n=24)$, ANA thealthy $(n=24)$ or SLE patients $(n=24)$. Single-cell analysis of cell surface markers was completed by mass cytometry on PBMCs and cellular heterogeneity was visualized using tSNE (figure 1A-B) and manual gating. Further, phospho-specific flow cytometry was used to measure basal levels of pERK, pPLCg2 and p38 and expression following CD3/CD28 (TCR) and anti-IgG and IgM (BCR) stimulation. Whole genome RNA-sequencing was performed on flow cytometry sorted T cells, B cells and monocytes from 35 matched ANA-, ANA +and SLE patients followed by weighted correlation network analysis (WGCNA) and pathway enrichment analyses.

Results Both European and African American SLE patients were distinguished from healthy individuals by $\mathrm{T}$ cell proliferation $(p=0.002)$ (figure $1 \mathrm{C}$ ), plasmacytoid dendritic cell activation $(p=0.021)$ and elevated stem cell factor $(p=0.0003)$. EA ANA+healthy individuals exhibited greater immune regulation with reduced $T$ cell numbers $(p=0.002)$ (figure $1 C)$, decreased activation of dendritic cells $(\mathrm{p}=0.039)$ and transitional $\mathrm{B}$ cells (0.033), and elevated expression of the inhibitory receptor CD85j $(p=0.042)$ on specific immune cell subsets compared to ANA- healthy subjects. Further, a module associated with hematopoiesis, $\mathrm{T}$ cell activation and intrinsic apoptosis signaling pathways is expressed at a higher level in $\mathrm{T}$ cells of EA ANA+individuals. In contrast, AA ANA+healthy individuals had elevated plasma levels of IL-6 $(p=0.018)$ and reduced inhibitory receptor expression $(p=0.0089)$ compared to ANAhealthy controls. Gene expression modules associated with viral responses and type I IFN pathway activation were identified in AA SLE patient B cells, while similar expression modules were only found in the monocytes of European American SLE patients.

Conclusions These results highlight the importance of stem cell factor and $\mathrm{T}$ cell expansion in SLE pathogenesis, and suggest that mechanisms of SLE pathogenesis differ by ethnicity. ANA +European Americans may have more effective regulatory mechanisms in place to prevent transition to classified autoimmune disease.

Funding Source(s): This work was supported by the NIH under award numbers U54GM104938,

U01AI101934, U19AI082714, and P30AR053483.

\section{CLINICIANS WITH LUPUS LOSING SKILLS AND DIVERSITY FROM HEALTH SERVICES RESULTS FROM AN ON-LINE SURVEY}

${ }^{1}$ Sara Booth*, ${ }^{2}$ Elizabeth Price, ${ }^{3}$ Elizabeth Walker. ${ }^{1}$ University of Cambridge; ${ }^{2}$ Hull York Medical School; ${ }^{3}$ Faculty of Medical Sciences

10.1136/lupus-2019-|sm.234

Background It is well-documented that people with systemic lupus erythematosus (SLE) have high levels of workplace disability and unemployment. We present the data from a sub-group of participants in an on-line survey designed to understand the barriers and facilitators to employment in people with lupus as a first step towards solutions. Three hundred and ninety-three detailed questionnaires were returned, demonstrating that the overarching barriers to continuing employment were fluctuation, invisibility and fatigue, facilitators were flexibility in working pattern, parttime working understanding management and colleagues. Participants suffered from high levels of anxiety and distress about their loss of income and likely future inability to work. Those claiming benefits felt guilt, shame and humiliation.

Methods The on-line survey was conducted in UK adults with SLE, through the LUPUS UK website, and was designed to find out about the difficulties and successes that people with SLE have in maintaining employment. The data from participants working in a clinical profession or employed by the National Health Service (NHS) in a clinicalrelated activity were identified from the cohort. Their data is currently being analysed to see if those working in NHS organisations have consistently good experiences of workplace support and better chances of retention.

Results Fifty-five of the sample of 393 (14\%) worked or had worked in the NHS in a clinical or clinically-related role. Roles include doctors, managers clinical coders, midwives, podiatrists, pharmacists, operating department assistants. The largest group were nurses including two in senior roles and untrained nursing assistants. Most of the sample had reduced hours or stopped working completely directly as a result of lupus, support was variable with some attributing workplace flexibility to NHS employment but others experiencing harassment. A recently introduced method of calculating sick leave militates against people with fluctuating conditions.

Conclusions SLE presents specific difficulties for maintaining employment fatigue, fluctuation and invisibility not addressed by current anti-discrimination legislation or currently-available reasonable adjustments. As Europes largest employer with a core mission of treating illness the NHS could set an example of how to overcome barriers to employment in people with fluctuating conditions, like lupus. There is clear evidence of loss of skills from the organisation and emerging evidence from this study suggest variable support and levels of knowledge form managers and colleagues. Personal pain from loss of role and income were high in participants. Further data will be available.

Funding Source(s): No external funding required

\section{IKZF1 AND IKZF3 INHIBITION IMPAIRS B CELL DIFFERENTIATION AND MODULATES GENE EXPRESSION IN SYSTEMIC LUPUS ERYTHEMATOSUS}

${ }^{1}$ Sotiria Manou-Stathopoulou*, ${ }^{1}$ Felice Rivellese, 'Daniele Mauro, 'Katriona Goldmann, ${ }^{2}$ Debasish Pyne, ${ }^{3}$ Peter Schafer, ${ }^{1}$ Michele Bombardieri, ${ }^{1}$ Costantino Pitzalis, ${ }^{1}$ Myles Lewis. ${ }^{1}$ Queen Mary University of London; ${ }^{2}$ Barts Health NHS Trust; ${ }^{3}$ Celgene Corporation

10.1136/lupus-2019-Ism.235

Background Polymorphisms in IKZF1 (Ikaros) and IKZF3 (Aiolos), which are transcriptions factors essential for $\mathrm{B}$ cell activation, maturation and differentiation, have been associated with Systemic Lupus Erythematosus (SLE). However the functional role of IKZF1 and IKZF3 in the context of failed B 